\title{
Effect of charge on graphene vacancy for hydrogen storage application
}

\author{
Gagus Ketut Sunnardianto*, Intan Ayu Larasati, Farid Triawan, and Ammar M. Aamer \\ Sampoerna University, Faculty of Engineering and Technology, Indonesia
}

\begin{abstract}
We investigated the effect of charge to the interaction between a hydrogen molecule and a hydrogenated vacancy $V_{11}$ in graphene surface based on density functional theory calculation. $V_{11}$ is graphene mono-vacancy with two hydrogen atoms adsorbed at the edge of vacancy. The hydrogen molecule physisorbed on deformed $\mathrm{V}_{11}$ is shown to dissociate producing a known stable vacancy $\mathrm{V}_{211}$, in which two carbon atoms are mono-hydrogenated and another is di-hydrogenated at the edge of the vacancy. We found that additional electron charge to the system could influence the reaction pathways and reduced the energy barrier for dissociation adsorption and desorption process provides a basic understanding in the mechanism of hydrogenation processes on graphene vacancy for hydrogen storage aplication.
\end{abstract}

\section{Introduction}

In recent years, immense attempts have been devoted to manage the graphene morphology affected by adsorbents, vacancy, and substrates. The morphology control is a key for functionalization of graphene. The chemical functionalization of pristine graphene sheet by absorbing hydrogen atoms attracts extensive attentions for controlling graphene physical properties. The functionality of hydrogenated graphene was predicted by Roman et al. [1] and Sofo et al. [2] by using density functional theory calculation. Hydrogenated graphene in the form of graphane was realized by Elias et al. [3] who demonstrated that the process of hydrogenation is reversible, making the hydrogenated graphene a potential candidate for hydrogen storage material. The advantage of graphene hydrogenation as a means for storing hydrogen [4]. Hydrogen is the lightest element and the most abundant element on the earth. Hydrogenation is simple adsorption and related with hydrogen storage [5]. Significant progresses have been made on the hydrogenation of graphene. Double sides hydrogenation bonded to the single atomic plane of graphite can tune the electronic properties of graphene [2]. Moreover, there is also much interest using graphene as an electrode material with widespread application such as, field-effect transistors [6], solar cells [7], supercapasitors [8], and batteries [9].

We have several observations on the hydrogenation graphene and graphene vacancy focusing on the reaction pathways and charge transfer rate from hydrogen to carbon on graphene $[4,5,10]$. However, we have not find yet an efficient method to reduce the energy barrier both of adsorption and desorption which is very important for hydrogen storage application.
We have reported the interaction between $\mathrm{H}_{2}$ and hydrogenated graphene vacancy $\left(\mathrm{V}_{11}\right)$ forming $\mathrm{V}_{211}$. We found the activation barrier of dissociation of $\mathrm{H}_{2}$ molecule on $\mathrm{V}_{11}$ is around $0.5 \mathrm{ev}$ [10]. However, we found the barrier height of hydrogen desorption from $\mathrm{V}_{211}$ to be $\mathrm{V}_{11}+\mathrm{H}_{2}$ is very hight around $3.01 \mathrm{eV}$. Thus, it is very difficult to desorp hydrogen atom from $V_{221}$ surface. Here, we would like to reduce the energy barrier of desorption process from $\mathrm{V}_{211}$ to be $\mathrm{V}_{11}+\mathrm{H}_{2}$. In this study, we would like to know the effect of charge (electron doping) to the reaction pathways and reduce the energy barrier of reversibe reaction of $\mathrm{V}_{11}+\mathrm{H}_{2}$ to be $\mathrm{V}_{211}$.

\section{Method}

We performed the structural and electronic structure optimization using density functional theory [11]. The simulations have been done using the quantum espresso (QE) code [12]. We used the projector-augmented- wave method and the ultra-soft pseudo potential [13]. The local density approximation by the Perdew-Zunger parameterization [14] was adopted. The parameters for hydrogenated graphene with a vacancy in a super cell were the energy cut off of $40 \mathrm{Ry}$ for the plane wave expansion of the wave function and 400 Ry for the expansion of the augmented charge. The convergence criterion for the structural optimization was that the total absolute value of the inter-atomic force vector became less than $10^{-4}$ Ry/a.u. The distance between graphene planes was separated by $10 \AA$ in orders to avoid interaction between layers. Distribution of k-points on a mesh of 12x12x1 was selected via Monkhrost-Pack scheme. The structure models used for simulation are graphene sheets consisting of 48 carbon atoms in rectangular super cells. The convergen check was done

\footnotetext{
* Corresponding author: gagus.sunardianto@sampoernauniversity.ac.id
} 
in a few times by tring the same simulations for a longer iteration numbers. In order to predict the reaction pathways and calculate the energy barrier, we derived potential energy surface by using NEB method.

\section{Results and Discussion}

\subsection{Reaction pathways of $\mathrm{H}_{2}$ dissociation on the $\mathrm{V}$ surface to form $\mathrm{V}_{11}\left(\mathrm{~V}+\mathrm{H}_{2}-\mathrm{V}_{11}\right)$}

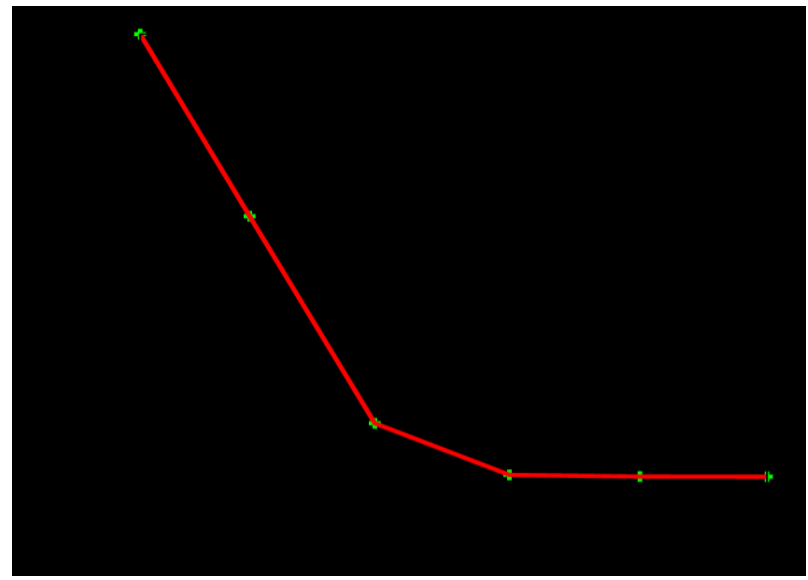

Fig. 1. A reaction pathway of dissoction of $\mathrm{H}_{2}$ on graphene vacancy surface.

Firstly, we investigated the $\mathrm{H}_{2}$ dissociation on the graphene vacancy surface. The simulations revealed that if a hydrogen molecule approaches a vacancy defect on a graphene surface, the molecule finally dissociates resulting $\mathrm{V}_{11}$ structure. There is no potential barrier in this reaction pathway (Fig. 1), suggesting that the dissociation of $\mathrm{H}_{2}$ on graphene vacancy surface could happens spontaneously due to the existence of dangling bonds acting as a reactive sites. Thus, hydrogen molecule can easily dissociate to form two hydrogen atoms and then adsorbed to the vacancy site resulting the $\mathrm{V}_{11}$ structure. Hydrogenated graphene vacancy experimentally revealed that $\mathrm{V}_{111}$ and $\mathrm{V}_{211}$ is the most stable of hydrogenated graphene vacancy [15]. Thus, we do hydrogenation again from resulted $\mathrm{V}_{11}$ structure. We exposed the $\mathrm{V}_{11}$ with one hydrogen molecule to form the most stable structure $\mathrm{V}_{211}$.

\subsection{Reaction pathways of $\mathrm{H}_{2}$ dissociation on the $V_{11}$ surface to form $V_{211}\left(V_{11}+H_{2} \rightarrow V_{211}\right)$}

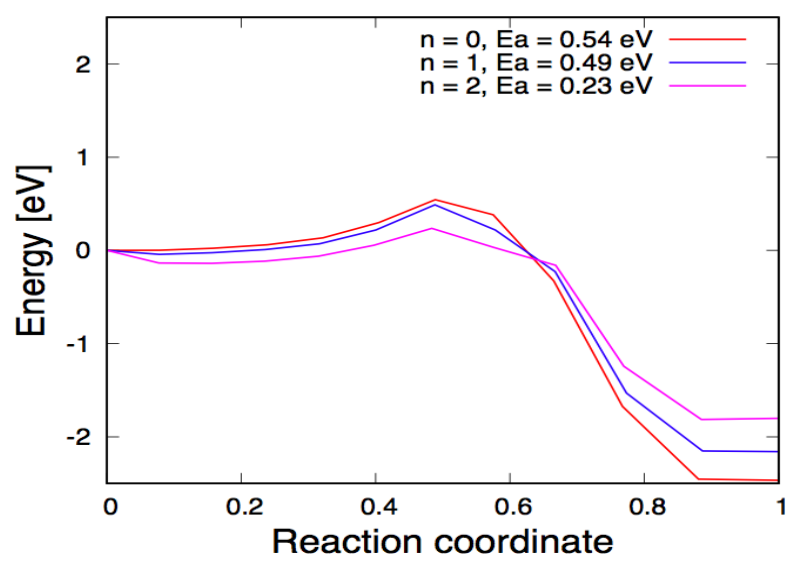

Fig. 2. A reaction pathway from $\mathrm{V}_{11}+\mathrm{H}_{2}$ to $\mathrm{V}_{211}$. In the inset, $\mathrm{n}=0$ means there is no additional charge to the system, $\mathrm{n}=1$ and $\mathrm{n}=2$ means one and two additional electron charge to the system.

Experimentally, mono-vacancy-hydrogen complexes in the single graphitic layer reveal that $\mathrm{V}_{111}$ and $\mathrm{V}_{211}$ are the stable structure [15]. Therefore we focus on the reaction of $\mathrm{V}_{11}+\mathrm{H}_{2}$ to form $\mathrm{V}_{211}$ as one of the most stable structures. After we find the reaction pathways of $\mathrm{H}_{2}+$ $\mathrm{V}_{11} \rightarrow \mathrm{V}_{211}$ [10]. We are now considerting the effect of charge (electron doping) on the reaction pathways and the energy barrier of the reversible reaction of $\mathrm{H}_{2}$ on $\mathrm{V}_{11}$ to form $V_{211}$ as shown in Fig. 2.

As you can see in Fig. 2 that one electron added to the system, it can reduces the energy barrier of hydrogen molecule dissociation-adsorption from $0.54 \mathrm{eV}$ to be $0.49 \mathrm{eV}$ and from $3.61 \mathrm{eV}$ to be $2.65 \mathrm{eV}$ for desorption process, subsequently two electron added to the system, it can reduces the energy barrier to be $0.23 \mathrm{eV}$ for dissociation-adsorption and to be $2.04 \mathrm{eV}$ for desorption. Thus, charging effect can reduce the energy barrier for both dissociation-adsorption and desorption process.

The novelty of this study, we found that the additional charge to the system could influence the reaction pathways of $\mathrm{H}_{2}$ dissociation on $\mathrm{V}_{11}$ surface to form $\mathrm{V}_{221}$ and its reversible reaction. Surpringly, we found that the effect of charge could reduce the energy barrier both for both adsoprtion and desorption. Thus, we found a new and simple method to reduce the energy barrier of dissociation-adsorption and desorption which is very important for hydrogen storage application.

\subsection{The proposed effective pathways of $\mathrm{H}_{2}$ dissociation on graphene vacancy for hydrogen storage application}

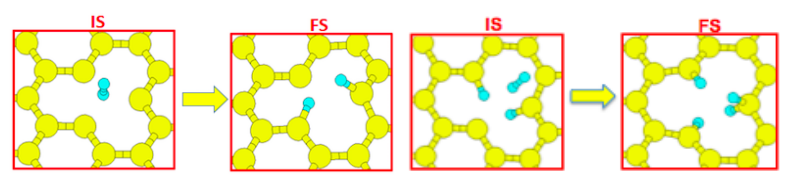

Fig 3. Structures of $\mathrm{V}+\mathrm{H}_{2} \rightarrow \mathrm{V}_{11}$ (left panel) and $\mathrm{V}_{11}+\mathrm{H}_{2} \rightarrow \mathrm{V}_{211}$ (right panel). The yellow and blue atoms represent carbon and hydrogen, respectively. Symbols IS and FS represent the initial and final state, respectively. 
once we found the reaction pathways of $\mathrm{V}+\mathrm{H}_{2} \rightarrow \mathrm{V}_{11}$ (Figure 1) and subsequently reaction pathways of $\mathrm{V}_{11}+\mathrm{H}_{2} \rightarrow \mathrm{V}_{211}$ (Figure 2) with additional charge to the system, we found the effective proposed reaction pathways for hydrogen storage application. As you can see in the Fig. 3 that in order to get the most stable structure of hydrogenated vacancy, we should start from the interaction of $\mathrm{H}_{2}$ on graphene vacancy $\left(\mathrm{V}+\mathrm{H}_{2} \rightarrow \mathrm{V}_{11}\right)$ and then subsequently, we exposed hydrogenated graphene vacancy with hydrogen molecule resulting hydrogenated graphene vacancy $\left(\mathrm{V}_{11}+\mathrm{H}_{2} \rightarrow \mathrm{V}_{211}\right)$. Thus, here we showed the proposed new pathway from the beginning of reaction $\left(\mathrm{V}+\mathrm{H}_{2} \rightarrow \mathrm{V}_{11}+\mathrm{H}_{2} \rightarrow \mathrm{V}_{211}\right)$ with charging effect as a new and simple method in reducing the activation barrier of $\mathrm{H}_{2}$ dissociation on graphene vacancy, which is very important for hydrogen storage application.

\section{Summary}

We have investigated the effect of charge on the interaction between hydrogen molecules on the $\mathrm{V}_{11}$ surface forming the most stable $\mathrm{V}_{211}$ structure by using Density Functional Theory calculation. We found a simple and easy method to reduce the energy barrier of both dissociation-adsorption and desorption by only adding electron (charging effect) on the system, which is very important for hydrogen storage application.

The calculations were carried out using the local computer at Sampoerna University and supported by supercomputer facilites of Graduate school of Engineering Science, Osaka University. GKS gratefully acknowledges to Prof. Koichi Kusakabe at Osaka University, Japan for his kind continuous support.

\section{References}

1. T. Roman, W. A. Dio, H. Nakanishi, H. Kasai, T. Sugimoto, K. Tange, Realizing a carbon-based hydrogen storage material, Jpn J Appl Phys, 45, 3A (2006)

2. J. O. Sofo, A. S. Chaudhari, G. D. Barber, Graphane: A two- dimensional hydrocarbon, Phys Rev B 75, 15 (2007)

3. D. C. Elias, R. R. Nair, T. M. G. Mohiuddin, S. V. Morozov, P. Blake, M. P. Halsall, A. C. Ferarri, D. W. Boukhvalov, M. I. Katsnelson, A. K. Geim, K. S. Novoselov, Control of graphene's properties by reversible hydrogenation: Evidence for graphane, Science, 323, 5914 (2009)

4. Sunnardianto GK, Maruyama I, Kusakabe K, Systematic study of the effect of $\mathrm{H}$ adsorption on the electron transfer rate in graphene, J. Comput. Theor. Nanosci 13 (2006)

5. Sunnardianto GK, Maruyama I, Kusakabe K, Storing-hydrogen processes on graphene activated by atomic-vacancies, Int. J. Hydrogen Energy 42 (2017)
6. B. Li, X. Cao, H. G. Ong, J. W. Cheah, X. Zhou, Z. Yin, H. Li, J. Wang, F. Boey, W. Huang, H. Zhang., All-carbon electronic devices fabricated by directly grown single-walled carbon nanotubues on reduced graphene oxide electrodes, Adv. Mater 22,28 (2010)

7. Y. Wang, X. Chen, Y. Zhong, F. Zhu, K. P. Loh., Large area,continuous, few-layered as anodes in organic photovoltaic devices, Appl. Phys. Lett 95 (2009)

8. Y. Zhu, S. Murali, M. D. Stoller, K. J. Ganesh, W. Cai, P. J. Ferreira, A. Pirkle, R. M. Wallace, K. A. Cychosz, M. Thommes, D. Su, E. A. Stach, R. S. Ruoff, Carbon-based supercapacitors produced by activa- tion of graphene science, Science $\mathbf{3 3 2}$ (2011)

9. M. L. Sanchez, P. Atienzar, G. Abelln, M. Puche, V. Forns, A. Ribera, H. Garca, The synthesis of a hybrid graphene-nickel/manganese mixed oxide and its performance in lithium-ion batteries, Carbon 50, 2, (2012)

10. Sunnardianto GK, Maruyama I, Kusakabe K. Dissociation chemisorption pathways of $\mathrm{H}_{2}$ molecule on graphene activated by a hydrogenated mono-vacancy $\mathrm{V}_{11}$. Adv. Sci. Eng. Med (2016)

11. R. M. Martin, Electronic Structure, Basic Theory, and Practical Methods, Cambridge University Press, (2004)

12. G. Kresse, J. Hafner, Ab initio molecular dynamics for liquid metals, Phys. Rev. B 47 (1993)

13. P. E. Bl“ochel, Projector augmented-wave method, Phys. Rev. B 50 (1994)

14. J. P. Perdew, A. Zunger, self-interaction correction to density-functional approximations for manyelectron systems, Phys. Rev. B 23 (1981)

15. Ziatdinov M, Fujii S, Kusakabe K, Kiguchi M, Mori T, Enoki T. Direct imaging of monovacancyhydrogen complexes in a single graphitic layer. Phys. Rev. B, 89, (2014) 